\title{
Personalidad internalizante y externalizante y efectos subjetivos en una muestra de adolescentes consumidores de cánnabis
}

\section{Internalizing and externalizing personality and subjective effects in a sample of adolescent cannabis users}

\author{
Sara García-Sánchez*, Josep Lluís Matalí*, María Martín-Fernández*, Marta Pardo*, \\ Maria lleras*, Carmina Castellano-Tejedor**, Josep Maria Haro***. \\ *Unidad de Conductas Adictivas. Servicio de Psiquiatría y Psicología de la infancia y la adolescencia. Hospital Sant Joan de \\ Déu. Barcelona CIBERSAM. **Departamento de Psiquiatría. Hospital Universitari Vall d'Hebron. Barcelona CIBERSAM. \\ ****Research Unit, Parc Sanitari Sant Joan de Déu, Sant Boi de Llobregat, Spain; Faculty of Medicine, Universitat de Barcelona, \\ Barcelona, Spain; CIBERSAM.
}

\section{Resumen}

El cánnabis es la sustancia ilícita más consumida por los adolescentes. Determinados rasgos de personalidad, como la impulsividad y la búsqueda de sensaciones, así como los efectos subjetivos experimentados tras el consumo (p.e. euforia o relajación), se han identificado como algunos de los principales factores etiológicos de consumo. Este estudio tiene por objetivo categorizar a una muestra de adolescentes consumidores de cánnabis en función de sus rasgos de personalidad más predominantes (perfil internalizante y externalizante) para, posteriormente, realizar una comparación de ambos perfiles a partir de un conjunto de variables asociadas al consumo, la gravedad clínica y los efectos subjetivos experimentados. A partir de un diseño transversal, se reclutaron 173 adolescentes (104 hombres y 69 mujeres) de 13 a 18 años, que demandaron tratamiento por Trastorno por Uso de Cánnabis en la Unidad de Conductas Adictivas (UCAD) del Hospital. Para la evaluación se utilizó un protocolo ad hoc para registrar el consumo, el Inventario Clínico para Adolescentes de Millon (MACI) y la versión abreviada del Addiction Research Center Inventory (ARCI)-49. El análisis factorial sugirió una solución en 2 perfiles: las escalas Introvertido, Inhibido, Pesimista, Histriónico (-), Egocéntrico (-), Autopunitivo y Tendencia límite forman el perfil internalizante, y las escalas Sumiso (-), Rebelde, Rudo, Conformista (-) y Oposicionista el externalizante. El análisis comparativo mostró que el perfil internalizante presenta mayores niveles de gravedad clínica y reporta más efectos subjetivos que el externalizante. Estos resultados sugieren la necesidad de diseñar estrategias de intervención específicas para cada perfil.

Palabras clave: Adolescentes; Cánnabis; Personalidad internalizante; Personalidad externalizante; Efectos subjetivos.

\begin{abstract}
Cannabis is the illicit substance most widely used by adolescents. Certain personality traits such as impulsivity and sensation seeking, and the subjective effects experienced after substance use (e.g. euphoria or relaxation) have been identified as some of the main etiological factors of consumption. This study aims to categorize a sample of adolescent cannabis users based on their most dominant personality traits (internalizing and externalizing profile). Then, to make a comparison of both profiles considering a set of variables related to consumption, clinical severity and subjective effects experienced. From a cross-sectional design, 173 adolescents (104 men and 69 women) aged 13 to 18 asking for treatment for cannabis use disorder in an Addictive Behavior Unit (UCAD) from the hospital were recruited. For the assessment, an ad hoc protocol was employed to register consumption, the Millon Adolescent Clinical Inventory (MACI) and the Addiction Research Center Inventory (ARCI) 49item short form were also administered. Factor analysis suggested a two-profile solution: Introverted, Inhibited, Doleful, Dramatizing (-), Egotistic (-), Self-demeaning and Borderline tendency scales composed the internalizing profile, and Submissive (-), Unruly, Forceful, Conforming (-) and Oppositional scales composed the externalizing profile. The comparative analysis showed that the internalizing profile has higher levels of clinical severity and more subjective effects reported than the externalizing profile. These results suggest the need to design specific intervention strategies for each profile.

Keywords: Adolescents; Cannabis; Internalizing personality; Externalizing personality; Subjective effects
\end{abstract}

Recibido: Septiembre 2015; Aceptado: Enero 2016.

Enviar correspondencia a:

Josep L. Matalí. Hospital Sant Joan de Déu, c/ Santa Rosa s/n, 08950 - Esplugues de Llobregat (Barcelona), España.

Teléfono: +34 9328040 00, Fax:+34 9360094 54. Email: jmatali@hsjdbcn.org 
$\mathrm{E}$ 1 cánnabis es la sustancia ilícita más consumida por los adolescentes a nivel mundial (United $\mathrm{Na}$ tion Office on Drugs and Crime, 2014). En España, una encuesta realizada a alumnos de entre 14 y 18 años revela que el $33.6 \%$ lo ha consumido alguna vez en su vida, el 26.6\% durante el último año, el $16.1 \%$ en los últimos 30 días y el $2.7 \%$ a diario, siendo los chicos más consumidores que las chicas (3.8\% vs. $1.5 \%)$ (Observatorio Español sobre Drogas, 2014).

Una edad de inicio de consumo precoz se relaciona con un mayor riesgo de consumo problemático (Martínez-Lorca y Alonso-Sanz, 2003), un uso posterior de otras drogas ilícitas (Swift et al., 2012), con la presencia de alteraciones cerebrales (DuPont y Lieberman, 2014; Jacobus y Tapert, 2014), del procesamiento cognitivo (Alameda-Bailén, Salguero-Alcañiz, Merchán-Clavellino, y Paíno-Quesada, 2014; Becker, Wagner, Gouzoulis-Mayfrank, Spuentrup, y Daumann, 2010; Grant, Chamberlain, Schreiber, y Odlaug, 2012) y de la función motora (Hall y Degenhardt, 2009), con la exacerbación de sintomatología psicopatológica en la edad adulta (Arias et al., 2013; Chen et al., 2011; Cuenca-Royo, Torrens, Sánchez-Niubó, Suelves, y Domingo-salvany, 2013; Muro i Rodríguez, 2015; Rubino, Zamberletti, y Parolaro, 2012) y con una mayor probabilidad de fracaso escolar (Volkow, Baler, Compton, y Weiss, 2014).

La etiología del consumo de cánnabis -esporádico o problemático- en población adolescente es multicausal (Hemphill et al., 2011), interactuando factores de riesgo individuales (Magallón-Neri et al., 2012; Szerman, Goti, Díaz, y Arango, 2014; ter Bogt et al., 2014), familiares (Becoña, Fernández del Río, Calafat, y Fernández-Hermida, 2014; Brière, Fallu, Descheneaux, y Janosz, 2011; Creemers et al., 2015), escolares (Guxens, Nebot, Ariza, y Ochoa, 2007; Hall y Degenhardt, 2009) y ambientales (Observatorio Europeo de las Drogas y las Toxicomanias, 2014; Peñafiel, 2009; Szerman et al., 2014).

Entre los factores de riesgo individuales destacan ciertos rasgos de personalidad (Belcher, Volkow, Moeller, y Ferré, 2014; Gunnarsson, Gustavsson, Tengström, Franck, y Fahlke, 2008; Marquez-Arrico y Adan, 2013) como altos niveles de impulsividad y búsqueda de sensaciones, rasgos disociales y baja predisposición a la evitación del daño (Gunnarsson et al., 2008; Munno, Saroldi, Bechon, Sterpone, y Zullo, 2015; Walther, Morgenstern, y Hanewinkel, 2012), que se agravarían con un consumo continuado (Chakroun, Doron, y Swendsen, 2004). También se han asociado con el consumo los déficits en la regulación emocional y la afectividad negativa (Chabrol, Melioli, y Goutaudier, 2014; Creemers et al., 2009). En estudios en los que se ha comparado a adolescentes consumidores y no consumidores utilizando el Inventario Clínico de Millon para Adolescentes (MACI) (Millon, 1993) se han observado que el grupo de consumidores presentaba puntuaciones más altas en las escalas de personalidad Rebelde, Rudo,
Oposicionista, Tendencia limite (Becoña et al., 2011; Fantin, 2006) y puntuaciones inferiores en las escalas de Sumiso y Conformista (Faúndez y Vinet, 2009). No obstante, los estudios con adolescentes son escasos, posiblemente, debido a la falta de consenso sobre la aplicabilidad del constructo de personalidad en edades tempranas o en la adolescencia (Adshead, Brodrick, Preston, y Deshpande, 2012).

Para facilitar el estudio de la personalidad algunos autores han sugerido englobarla en las dimensiones de internalización y externalización (Achenbach y Edelbrock, 1984) y las investigaciones indican que éstas explican la mayor parte de las asociaciones entre patrones de personalidad, indicadores de psicopatología y disfunción clínica en pacientes (Cosgrove et al., 2011; Harford et al., 2013; Hink et al., 2013; Hopwood y Grilo, 2010).

Por otra parte, diversos estudios determinan que el uso problemático del cánnabis también estaría relacionado con la experiencia subjetiva que se obtenga con el consumo (Zeiger et al., 2010). Los efectos subjetivos se caracterizan en dos subtipos: 1) los efectos positivos; vinculados a experiencias agradables como la euforia, la relajación, o la alteración sensorial, y 2) los efectos negativos; ligados a experiencias desagradables como ansiedad, paranoia, alucinaciones, tristeza o náuseas. Los usuarios de cánnabis pueden reportar ambos (Block, Erwin, Farinpour, y Braverman, 1998; Scherrer et al., 2009; Zeiger et al., 2012). Dichos efectos han demostrado ser buenos predictores del abuso o dependencia del cánnabis (Pedrero Pérez, 2003; Zeiger et al., 2012). En población adolescente se ha observado que las experiencias subjetivas positivas antes de los 16 años están relacionadas con dependencia al cánnabis en la edad adulta (Fergusson, Horwood, Lynskey, y Madden, 2003). Más recientemente, se ha observado que experimentar efectos subjetivos positivos y negativos, denominado como "alta respuesta", está vinculado a la tendencia para consumir y desarrollar dependencia al cánnabis (Scherrer et al., 2009) y a la presencia de mayores tasas de consumo de otras sustancias ilegales (Zeiger et al., 2012).

Vista la importancia de los rasgos de personalidad en el debut y el mantenimiento del consumo y de los efectos subjetivos en el desarrollo de un uso problemático del cánnabis, así como los pocos datos existentes al respecto, se presenta este estudio con los siguientes objetivos. En primer lugar, discriminar los rasgos de personalidad presentes en una muestra clínica de adolescentes consumidores de cánnabis a través de la categorización en dos perfiles de personalidad: internalizante y externalizante. En segundo lugar, realizar una comparación de ambos perfiles mediante un conjunto de variables asociadas al consumo tales como: género, edad de inicio de consumo, edad de consumo regular, edad de demanda de tratamiento, patrón horario de consumo, gravedad clínica y los efectos subjetivos experimentados tras el consumo. 


\section{Método}

El diseño del presente trabajo es exploratorio de tipo transversal.

\section{Participantes}

Para llevar a cabo la presente investigación, se reclutó a un total de 173 adolescentes que acudieron a la Unidad de Conductas Adictivas (UCAD) del Servicio de Psiquiatría y Psicología del Hospital (anonimizado para revisión por pares) con demanda de tratamiento por consumo de cánnabis.

Los criterios de inclusión de la muestra fueron: 1) tener una edad inferior a 18 años y 2) presentar criterios de abuso o dependencia al cánnabis. Por otra parte, el criterio de exclusión fue: 1) presencia de patología mental aguda que impidiera la comprensión de los cuestionarios.

La muestra final se compuso de un total de 173 sujetos; $60.1 \%(\mathrm{n}=104)$ hombres y $39.9 \%(\mathrm{n}=69)$ mujeres, con una edad media de 15.67 años $(\mathrm{DE}=1.23)$.

En relación al ámbito académico, de los 173 sujetos, el $70.5 \%(\mathrm{n}=122)$ estaba estudiando, el $69.4 \%(\mathrm{n}=120)$ había repetido curso y el $29.5 \%(\mathrm{n}=51)$ había abandonado los estudios. En el momento de la evaluación los participantes cursaban o habían cursado; el $13.3 \%(n=23) 2^{\circ}$ de ESO, el $27.7 \%(\mathrm{n}=48) 3^{\circ} \mathrm{de}$ ESO, el $31.2 \%(\mathrm{n}=54) 4^{\circ} \mathrm{de}$ ESO, el $8.7 \%(\mathrm{n}=15) 1^{\circ}$ de Bachillerato, el $0.6 \%(\mathrm{n}=1)$ $2^{\circ}$ de Bachillerato, el $8.1 \%(n=14)$ Programas de Calificación Profesional Inicial (PCPI), el $0.6 \%(\mathrm{n}=1)$ estudios universitarios y el $9.8 \%(\mathrm{n}=17)$ otro tipo de formaciones académicas.

\section{Instrumentos de evaluación}

Para recabar la información necesaria para la investigación se recogieron las variables socio-demográficas de género, edad y nivel académico. Las variables relacionadas con el consumo de cánnabis se obtuvieron mediante un protocolo ad hoc en el que se registró la edad de inicio del consumo, la edad de consumo regular, la edad de demanda de tratamiento y el patrón horario de consumo (antes de entrar a clase, en el patio, al medio día, por las tardes, antes de ir a dormir).

Para determinar la existencia de Trastorno por Uso de Sustancias se usó la versión española del Suplemento de Abuso de sustancias y otros trastornos de la entrevista diagnóstica semi-estructurada Kiddie-Schedule for Affective Disorders $\mathcal{E}$ Schizophrenia, Present $\mathcal{E}$ Lifetime Version (K-SADS-PL); (Kaufman et al., 1997; Ulloa et al., 2006) que se fundamenta en los criterios del DSM-IV. La K-SADS-PL está diseñada para evaluar episodios de psicopatología en niños y adolescentes (de 6 a 17 años) en el pasado y en la actualidad. Los coeficientes de fiabilidad de la versión española de la escala van de 0.76 para Trastorno Depresivo mayor a 1 para Trastorno Disocial.

Para evaluar los patrones de personalidad y la gravedad clínica de los pacientes se utilizó la versión española del In- ventario Clínico para Adolescentes de Millon (Millon Adolescent Clinical Inventory, MACI); (Millon, 1993). El MACI es un cuestionario diseñado para evaluar las características de personalidad en adolescentes (de 13 a 19 años), que consta de 160 ítems con formato de respuesta verdadero-falso y se organiza en 31 escalas, 27 clínicas y 4 no clínicas. Éstas son: Doce escalas de patrones de personalidad que revelan los estilos de personalidad que surgen durante el desarrollo infantil y se estabilizan en la adolescencia (Inhibido, Pesimista, Sumiso, Histriónico, Egocéntrico, Rebelde, Rudo, Conformista, Oposicionista, Autopunitivo y Tendencia Limite). Ocho escalas de preocupaciones expresadas que se focalizan en áreas del desarrollo adolescente (Difusión de la identidad, Desvalorización de si mismo, Desagrado con el propio cuerpo, Incomodidad respecto al sexo, Inseguridad con los iguales, Insensibilidad social, Discordancia familiar y Abusos en la infancia). Siete escalas de síndromes clínicos, que involucran trastornos de alta prevalencia en los adolescentes (Trastornos de la alimentación, Inclinación al abuso de sustancias, Predisposición a la delincuencia, Propensión a la impulsividad, Sentimientos de ansiedad, Afecto Depresivo y Tendencia al suicidio). Una escala de validez del protocolo (Fiabilidad) y tres escalas modificadoras que permiten controlar ciertas tendencias de respuesta (Transparencia, Deseabilidad y Alteración); (Faúndez y Vinet, 2009). Este instrumento se validó en población española y los coeficientes de fiabilidad de las escalas se situaban entre 0.65 (Incomodidad respecto al sexo) y 0.91 (Desvalorización de sí mismo). Entre las escalas de patrones de personalidad, los coeficientes van de 0.69 (Sumiso) a 0.90 (Autopunitivo); (Millon y Aguirre, 2004). En la muestra del presente estudio, el coeficiente de fiabilidad para todos los ítems se situó en 0.66 .

Para estudiar los efectos subjetivos que experimentan los adolescentes tras el consumo de cánnabis se administró la versión española abreviada del Addiction Research Center Inventory (ARCI)-49 (Martin, Sloan, Sapira, y Jasinski, 1971), por ser uno de los cuestionarios más utilizados en el campo de los ensayos clínicos de sustancias con potencial de abuso (Poudevida, Farré, Roset, y Camí, 2003). Este cuestionario consta de 49 ítems de formato verdadero-falso y se compone de 5 escalas que diferencian 5 tipos de efectos subjetivos: 1) la escala Grupo Morfina-Bencedrina (BMG) que mide el efecto de Euforia, 2) la escala Grupo Pentobarbital-Clorpromazina-Alcohol (PCAG) que mide el efecto de $S e$ dación, 3) la escala Dietilamida del Ácido Lisérgico (LSD) que mide los efectos de Disforia y los cambios psicotomiméticos, 4) la escala Grupo Bencedrina (BG) que mide la Eficiencia intelectualy 5) la escala Grupo Anfetamina (A) mide el efecto Anfetaminico.

Este instrumento se validó en población española y los coeficientes de fiabilidad de las escalas eran de 0.87 para la escala PCAG; 0.81 para MBG, 0.55 para LSD, 0.79 para BG y 0.49 para A (Lamas, Farré, Llorente, y Camí, 1994). En la muestra del presente estudio, el coeficiente de fiabilidad para los 49 ítems se situó en 0.78. 


\section{Procedimiento}

Esta investigación se llevó a cabo íntegramente en la Unidad de Conductas Adictivas de adolescentes del Hospital (anonimizado para revisión por pares).

Previamente a la realización del trabajo, se obtuvo la aprobación del Comité de Ética hospitalario -considerando la normativa ética interna, así como la de la Asociación Médica Mundial y la Declaración de Helsinki del 1975 con sus sucesivas enmiendas (CIOMS y WHO, 1993) y todos los participantes otorgaron su consentimiento por escrito tras recibir la información relativa al trabajo, sus objetivos y al acuerdo de confidencialidad y protección de datos personales. La participación en este estudio no fue remunerada.

Todos los participantes fueron evaluados por el psicólogo clínico de la unidad durante 2 sesiones de 45 minutos de duración aproximadamente. En la primera sesión se realizó la anamnesis psicológica, se recogieron los datos socio-demográficos y el registro del consumo (protocolo ad hoc). En la segunda sesión se administraron los cuestionarios MACI y ARCI-49 a los adolescentes y, posteriormente, se revisaron para comprobar que estuvieran debidamente cumplimentados.

\section{Análisis estadístico}

Para el tratamiento estadístico de los datos se utilizó el Statistical Package for the Social Sciences (SPSS), en su versión 18 (SPSS Inc., 1988).

Para analizar la prevalencia de las distintas características socio-demográficas y las variables asociadas al consumo consideradas, se realizaron análisis descriptivos y de distribución de frecuencias.

A continuación, se realizó una categorización de las escalas de patrones de personalidad del MACI en perfiles mediante un análisis factorial por el método de extracción de componentes principales y rotación VARIMAX en el que se obtuvieron los perfiles internalizante/externalizante. Una vez obtenidos, la muestra se distribuyó en ambos perfiles mediante un análisis de conglomerado $k$ medias y se analizaron las diferencias entre éstos en función de las variables socio-demográficas y las variables relacionadas con el consumo. Para ello, se ha realizado una comparación de medias mediante la prueba de $t$ de Student para muestras independientes (para las variables cuantitativas) y la prueba de Chi cuadrado, calculada a partir de tablas de contingencia 2X2 (para las variables nominales).

La significación de todas las pruebas se ha considerado con un nivel de probabilidad del $5 \%$ o inferior, indicando siempre la significación exacta que ofrecía el SPSS.

\section{Resultados}

\section{Descriptivos de la muestra}

En relación al consumo de cánnabis, la media de edad de inicio de consumo fue de 13.01 años $(D E=1.63)$. La me- dia de edad de consumo regular fue de $13.80(D E=1.51)$. La media de edad de demanda de tratamiento fue de 15.67 años $(D E=1.23)$.

En relación al patrón horario de consumo, del total de la muestra $(n=173)$, un $63.6 \%(n=110)$ fuman antes de entrar a la escuela, un $54.3 \%(n=94)$ fuman durante el patio, un $64.7 \%$ ( $n=112)$ fuman al salir de clase al mediodía, un $69.9 \%(n=121)$ fuman por la tarde y un $31.8 \%(n=55)$ fuman antes de ir a dormir.

\section{Análisis factorial exploratorio del MACI}

Se ha llevado a cabo un análisis factorial exploratorio (AFE) para determinar si las escalas del Patrón de Personalidad del MACI se podían representar mediante una estructura de dos perfiles: internalizante y externalizante (Hopwood y Grilo, 2010; Newman, Larsen, Cunningham, y Barry, 2015).

La medida de adecuación muestral Kaiser-Meyer-Olkin indicó que la relación entre las escalas del Patrón de personalidad del MACI es notable $(\mathrm{KMO}=.856)$, y la prueba de esfericidad de Bartlett señaló que se puede aplicar el análisis factorial $\left(X^{2}=1902.944, p<.001\right)$.

El Análisis de Componentes Principales (ACP) indicó 2 factores con un valor propio superior a 1 (5.356, 3.732). Se extrajo un modelo de dos factores que explica el $75.73 \%$ de la varianza; un $44.12 \%$ con el primer componente y un $31.62 \%$ con el segundo.

La matriz de componentes rotados (Varimax) sugirió la siguiente agrupación. El perfil internalizante está compuesto por las escalas: Introvertido, Inhibido, Pesimista, Histriónico (signo negativo), Egocéntrico (signo negativo), Autopunitivo, Tendencia límite. El perfil externalizante lo componen las escalas: Sumiso (signo negativo), Rebelde, Rudo, Conformista (signo negativo) y Oposicionista. Las cargas negativas de las

Tabla 1. Análisis Factorial Exploratorio de las escalas de Patrón de personalidad del $\mathrm{MACl}$.

\begin{tabular}{lcc}
\hline Escalas & \multicolumn{2}{c}{ Componente } \\
\cline { 2 - 3 } & Internalizante & Externalizante \\
\hline Histriónico (4) & -.864 & \\
Introvertido (1) & .844 & \\
Egocéntrico (5) & -.841 & \\
Autopunitivo (8B) & .835 & \\
Pesimista (2B) & .832 & \\
Inhibido (2A) & .789 & \\
Tendencia límite (9) & .692 & .863 \\
Rebelde (6A) & & .846 \\
Rudo (6B) & & -.843 \\
Sumiso (3) & & -.795 \\
Conformista (7) & & .647 \\
Oposicionista (8A) & & \\
\hline
\end{tabular}


escalas Histriónico y Egocéntrico sugieren que puntuar bajo en éstas determina la pertenencia al perfil internalizante. Del mismo modo, las cargas negativas en las escalas sumiso y conformista señalan que puntuar bajo determina la pertenencia al perfil externalizante (ver Tabla 1).

\section{Análisis de conglomerado de $\mathbf{k}$ medias}

A partir de un análisis de conglomerado de $k$ medias hemos clasificado a la muestra en los dos perfiles obtenidos en el análisis factorial (internalizante y externalizante). En el conglomerado 1, perfil internalizante, se agrupan el $49.1 \%$ de los participantes $(n=85)$. El conglomerado 2, perfil externalizante, lo forman el $50.1 \%$ de los participantes $(n=$ 88). El análisis de la varianza de un factor (ANOVA) indica que todas las escalas son significativamente diferentes entre los conglomerados, excepto Rudo $(p=.488)$ que es la escala en la que son más similares ambos conglomerados.

\section{Análisis comparativo entre perfiles internalizante/ externalizante}

Para las variables: edad de inicio de consumo, edad de consumo regular y edad de demanda de tratamiento, no existen diferencias estadísticamente significativas entre perfiles internalizante y externalizante (ver Tabla 2).

En relación al género, del total de internalizantes $(n=$ $85)$, el $58.8 \%(n=50)$ son hombres y el $41.2 \%(n=35)$ son mujeres. De todos los externalizantes $(n=88)$, el $61.4 \%(n$ $=54)$ son hombres frente al $38.6 \%(n=34)$ que son mujeres. No existen diferencias estadísticamente significativas entre perfiles en función del género.

Tabla 2. Comparativa entre perfiles en función de la edad de inicio, de consumo regular y de demanda de tratamiento.

\begin{tabular}{|c|c|c|c|c|c|c|c|c|}
\hline \multirow[t]{2}{*}{ Edad } & \multirow{2}{*}{$\begin{array}{c}\text { Muestra total } \\
(N=173) \\
\text { Media }\end{array}$} & \multirow{2}{*}{$\begin{array}{c}\begin{array}{c}\text { Internalizante } \\
(n=85)\end{array} \\
\mathrm{DE} \\
\end{array}$} & \multicolumn{2}{|c|}{$\begin{array}{l}\text { Externalizante } \\
\quad(n=88)\end{array}$} & \multirow[b]{2}{*}{ Media } & \multirow[b]{2}{*}{$\mathrm{DE}$} & \multirow[b]{2}{*}{$\mathrm{t}$} & \multirow[b]{2}{*}{$p$} \\
\hline & & & Media & $\mathrm{DE}$ & & & & \\
\hline $\begin{array}{l}\text { Edad de inicio } \\
\text { de consumo }\end{array}$ & 13.01 & 1.63 & 13.02 & 1.57 & 13 & 1.69 & -.533 & n.s. \\
\hline $\begin{array}{l}\text { Edad de consumo } \\
\text { regular }\end{array}$ & 13.80 & 1.51 & 13.74 & 1.48 & 13.86 & 1.53 & .095 & n.s. \\
\hline $\begin{array}{l}\text { Edad de demanda } \\
\text { de tratamiento }\end{array}$ & 15.67 & 1.23 & 15.76 & 1.21 & 15.58 & 1.24 & .986 & n.s. \\
\hline
\end{tabular}

Nota. n.s.: diferencias estadísticamente no significativas según la t de Student ( $p>$.05).

Tabla 3. Comparativa entre perfiles en función del patrón horario de consumo.

\begin{tabular}{|c|c|c|c|c|c|c|c|c|}
\hline \multirow[t]{2}{*}{ Patrón horario } & \multirow{2}{*}{$\begin{array}{c}\begin{array}{c}\text { Muestra total } \\
(N=173)\end{array} \\
n\end{array}$} & \multirow{2}{*}{$\begin{array}{c}\begin{array}{c}\text { Internalizante } \\
(\boldsymbol{n}=\mathbf{8 5})\end{array} \\
\%\end{array}$} & \multicolumn{2}{|c|}{$\begin{array}{l}\text { Externalizante } \\
\quad(n=88)\end{array}$} & \multirow[b]{2}{*}{$\mathrm{n}$} & \multirow[b]{2}{*}{$\%$} & \multirow[b]{2}{*}{ Chi Cuadrado } & \multirow[b]{2}{*}{$\mathrm{p}$} \\
\hline & & & $\mathrm{n}$ & $\%$ & & & & \\
\hline \multicolumn{9}{|l|}{$\begin{array}{l}\text { Antes de } \\
\text { entrar a clase }\end{array}$} \\
\hline 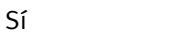 & 110 & 63.6 & 53 & 62.4 & 57 & 64.8 & & \\
\hline No & 63 & 36.4 & 32 & 37.6 & 31 & 35.2 & .109 & n.s. \\
\hline Sí & 94 & 50.3 & 42 & 49.4 & 50 & 59.1 & & \\
\hline No & 79 & 45.7 & 43 & 50.6 & 36 & 40.9 & 1.633 & n.s. \\
\hline \multicolumn{9}{|l|}{ Al mediodía } \\
\hline Sí & 112 & 64.7 & 56 & 65.9 & 56 & 63.6 & & \\
\hline No & 61 & 35.3 & 29 & 34.1 & 32 & 36.4 & .096 & n.s. \\
\hline \multicolumn{9}{|l|}{ Por la tarde } \\
\hline Sí & 121 & 69.9 & 62 & 72.9 & 59 & 67 & & \\
\hline \multicolumn{9}{|l|}{ Antes de dormir } \\
\hline Sí & 55 & 31.8 & 34 & 40 & 21 & 23.9 & & \\
\hline No & 118 & 68.2 & 51 & 60 & 67 & 76.1 & 5.192 & .023 \\
\hline
\end{tabular}

Nota. n.s.: diferencias estadísticamente no significativas según la Chi Cuadrado ( $\mathrm{p}>$.05). 
Tabla 4. Comparativa entre perfiles en función de las escalas de preocupaciones expresadas y síndromes clínicos del MACl.

\begin{tabular}{|c|c|c|c|c|c|c|c|c|}
\hline MACI & $\begin{array}{l}\text { Muestra total } \\
\qquad(\mathrm{N}=173)\end{array}$ & $\begin{array}{l}\text { Internalizante } \\
\quad(n=85)\end{array}$ & $\begin{array}{l}\text { Externalizante } \\
\qquad(\mathrm{n}=\mathbf{8 8})\end{array}$ & & & & & \\
\hline & Media & $\mathrm{DE}$ & Media & $\mathrm{DE}$ & Media & $\mathrm{DE}$ & $\mathrm{T}$ & $\mathrm{p}$ \\
\hline $\begin{array}{l}\text { Desvalorización de } \\
\text { sí mismo }\end{array}$ & 48.33 & 20.54 & 62.45 & 16.26 & 34.69 & 14.01 & 12.038 & $<.001$ \\
\hline $\begin{array}{l}\text { Desagrado por el } \\
\text { propio cuerpo }\end{array}$ & 47.80 & 22.13 & 58.78 & 23.45 & 37.19 & 14.37 & 7.330 & $<.001$ \\
\hline $\begin{array}{l}\text { Incomodidad } \\
\text { respecto al sexo }\end{array}$ & 35.18 & 23.95 & 34.45 & 25.18 & 35.89 & 22.83 & -.394 & n.s. \\
\hline $\begin{array}{l}\text { Inseguridad con los } \\
\text { iguales }\end{array}$ & 38.39 & 21.54 & 47.95 & 22.22 & 29.15 & 16.27 & 6.365 & $<.001$ \\
\hline Insensibilidad social & 79.11 & 25.82 & 68.21 & 26.31 & 89.64 & 20.54 & -5.981 & $<.001$ \\
\hline $\begin{array}{l}\text { Abusos en la } \\
\text { infancia }\end{array}$ & 57.21 & 21.07 & 67.68 & 19.41 & 47.10 & 17.43 & 7.342 & $<.001$ \\
\hline $\begin{array}{l}\text { Trastorno de la } \\
\text { alimentación }\end{array}$ & 48.95 & 20.36 & 58.25 & 19.72 & 39.98 & 16.68 & 6.586 & $<.001$ \\
\hline $\begin{array}{l}\text { Inclinación al abuso } \\
\text { de sustancias }\end{array}$ & 86.69 & 17.66 & 90.12 & 16.85 & 83.39 & 17.88 & 2.545 & .012 \\
\hline $\begin{array}{l}\text { Predisposición a la } \\
\text { delincuencia }\end{array}$ & 83.87 & 22.96 & 76.49 & 23.51 & 90.99 & 20.09 & -4.363 & $<.001$ \\
\hline $\begin{array}{l}\text { Propensión a la } \\
\text { impulsividad }\end{array}$ & 76.94 & 25.33 & 76.60 & 24.17 & 77.26 & 26.52 & -.171 & n.s. \\
\hline $\begin{array}{l}\text { Sentimientos de } \\
\text { ansiedad }\end{array}$ & 21.43 & 16.81 & 22.95 & 18.84 & 19.95 & 14.54 & 1.174 & n.s. \\
\hline
\end{tabular}

Nota. n.s.: diferencias estadísticamente no significativas según la t de Student ( $p$ > .05).

En relación a los patrones horarios de consumo, de los externalizantes, el $64.8 \%(n=57)$ fuman antes de entrar a clase y el $59.1 \%(n=52)$ a la hora del patio vs. el $62.4 \%$ $(n=53)$ y el $49.4 \%(n=42)$ de internalizantes, respectivamente. De igual manera, al salir de la escuela al mediodía el $65.9 \%(n=56)$ de los internalizantes fuman vs. el $63.6 \%$ $(n=56)$ de los externalizantes. De todos los internalizantes $(n=85)$, el $72.9 \%(n=62)$ fuman por la tarde vs. el $67 \%(n=59)$ de externalizantes. No obstante, no existen diferencias estadísticamente significativas entre perfiles para ninguna de estas cuatro variables. Por el contrario, el $40 \%(n=34)$ de los internalizantes fuman antes de dormir vs. el $23.9 \%(n=21)$ de los externalizantes, siendo ésta diferencia estadísticamente significativa $(p=.023)$ (ver Tabla 3).

\section{Preocupaciones expresadas (MACI)}

Para la categoría de preocupaciones expresadas del MACI, existen diferencias estadísticamente significativas entre perfiles puntuando los internalizantes significativamente más alto que los externalizantes en las escalas: Difusión de la identidad $\left(t_{(171)}=6.197, p<.001\right.$, IC $95 \% 13.21-25.56, d$
$=1)$, Desvalorización de sí mismo $\left(t_{(171)}=12.038, p<.001, I C\right.$ $95 \% 23.20-32.30, d=1.8)$, Desagrado con el propio cuerpo $\left(t_{(171)}=7.330, p<.001, I C 95 \% 15.77-27.39, d=1.1\right)$, Inseguridad con los iguales $\left(t_{(171)}=6.365, p<.001\right.$, IC $95 \% 12.97-$ $24.63, d=0.9)$ y Abusos en la infancia $\left(t_{(171)}=7.342, p<.001\right.$, IC $95 \% 15.04-26.11, d=1.1)$.

Del mismo modo, existen diferencias estadísticamente significativas entre perfiles siendo los externalizantes los que puntúan significativamente más alto que los internalizantes en la escala: Insensibilidad social $\left(t_{(171)}=-5.981, p<\right.$ .001$, IC $95 \%-28.49--14.35, d=0.9)$.

En las escalas Incomodidad respecto al sexo y Discordancia familiar no se observan diferencias estadísticamente significativas entre perfiles internalizante y externalizante (ver Tabla 4).

\section{Síndromes clínicos (MACI)}

Para la categoría de sindromes clínicos del MACI se observan diferencias estadísticamente significativas entre perfiles a favor de los internalizantes que puntúan significativamente más alto que los externalizantes en las escalas: Trastornos de la alimentación $\left(t_{(171)}=6.586, p<.001\right.$, IC $95 \%$ 
Tabla 5. Comparativa entre perfiles en función de las escalas de Efectos subjetivos del ARCI.

\begin{tabular}{|c|c|c|c|c|c|c|c|c|}
\hline $\mathrm{ARCl}$ & $\begin{array}{l}\text { Muestra total } \\
\qquad(\mathrm{N}=173)\end{array}$ & $\begin{array}{l}\text { Internalizante } \\
\quad(n=85)\end{array}$ & & & & & & \\
\hline & Media & $\mathrm{DE}$ & Media & $\mathrm{DE}$ & Media & $\mathrm{DE}$ & $\mathrm{T}$ & $\mathrm{p}$ \\
\hline Sedación & 3.54 & 3.37 & 4.34 & 3.66 & 2.78 & 2.89 & 3.103 & .002 \\
\hline Euforia & 7 & 3.17 & 7.34 & 3.24 & 6.65 & 3.08 & 1.405 & n.s. \\
\hline $\begin{array}{l}\text { Disforia/ } \\
\text { Efectos físicos } \\
\text { desagradables }\end{array}$ & 0.89 & 2.50 & 1.31 & 2.57 & 0.49 & 2.38 & 2.155 & .033 \\
\hline $\begin{array}{l}\text { Eficiencia } \\
\text { intelectual }\end{array}$ & 1.47 & 2.87 & 1.49 & 3.35 & 1.44 & 2.30 & 0.107 & n.s. \\
\hline $\begin{array}{l}\text { Efectos } \\
\text { anfetamínicos }\end{array}$ & 4.45 & 1.95 & 4.63 & 2.01 & 4.27 & 1.89 & 1.204 & n.s. \\
\hline
\end{tabular}

Nota. n.s.: diferencias estadísticamente no significativas según la t de Student ( $p>$.05).

$12.79-23.74, d=1.08)$, Inclinación al abuso de sustancias $t_{(171)}=2.545, p=0.012$, IC $\left.95 \% 1.51-11.95, d=0.4\right)$, Afecto Depresivo $\left(t_{(171)}=11.523, p<.001\right.$, IC $95 \% 23.471-33.174, d$ $=1.8)$ y Tendencia al suicidio $\left(t_{(171)}=8.729, p<.001\right.$, IC $95 \%$ $15.42-24.44, d=1.3)$.

A su vez, existen diferencias estadísticamente significativas entre perfiles donde los externalizantes puntúan significativamente más alto que los internalizantes en la escala: Predisposición a la delincuencia $\left(t_{(171)}=-4.363, p<.001, I C\right.$ $95 \%-21.05--7.93, d=0.65)$.

En las escalas Propensión a la impulsividady Sentimientos de Ansiedad no se observan diferencias estadísticamente significativas entre perfiles internalizante y externalizante (ver Tabla 4).

\section{Efectos subjetivos (ARCI)}

Respecto a los efectos subjetivos del consumo del ARCI, se encuentran diferencias significativas entre perfiles en las que los internalizantes obtienen puntuaciones significativamente mayores que los externalizantes en las escalas que miden los efectos de: Sedación (PCAG) $\left(t_{(169)}=3.103, p=\right.$ $.002, I C 95 \% 0.56-2.55, d=0.78)$ y Disforia (LSD) $\left(t_{(167)}=\right.$ $2.155, p=.033$, IC $95 \% 0.06-1.57, d=0.5)$.

En las escalas que miden los efectos de Euforia (BMG), Eficiencia intelectual (BG) y los de tipo Anfetamínico (A) no se observan diferencias estadísticamente significativas entre perfiles internalizante y externalizante (ver Tabla 5 ).

\section{Discusión}

Este estudio se ha llevado a cabo para discriminar los rasgos de personalidad presentes en una muestra de adolescentes consumidores de cánnabis, a través de una categorización en perfiles internalizante y externalizante para, posteriormente, realizar una comparación entre ambos perfiles en función de variables relacionadas con el consumo, gravedad clínica y efectos subjetivos.
Los resultados de la presente investigación apoyan la existencia de diferencias significativas en los rasgos de personalidad de los adolescentes consumidores de cánnabis. Se han obtenido dos perfiles de personalidad, internalizante y externalizante, que fundamentan el constructo dimensional de la personalidad en adolescentes consumidores de cánnabis (Hopwood y Grilo, 2010). Cabe señalar que la distribución de la muestra entre perfiles fue equilibrada, ya que el número de participantes que incluyó cada perfil resultó similar y, por tanto, los resultados parecen señalar que en una muestra clínica de adolescentes consumidores de cánnabis no predomina un perfil sobre otro.

Los resultados obtenidos muestran que los adolescentes con un perfil internalizante se caracterizan por mostrar mayores puntuaciones en las escalas Introvertido, Inhibido, Pesimista, Autopunitivo, Tendencia Límitey puntuaciones muy bajas en las escalas Histriónico y Egocéntrico. Por su parte, los adolescentes con un perfil externalizante alcanzan puntuaciones mayores en las escalas Rebelde, Rudo, Oposicionista y puntuaciones muy bajas en Conformista y Sumiso (Hopwood y Grilo, 2010). No se observaron diferencias estadísticamente significativas entre perfiles de personalidad en función del género. Éste hecho también se observó en el estudio de Hopwood y Grilo y se podría explicar en base a que ambos trabajos están realizados con muestras clínicas, dónde la comorbilidad con trastornos externalizantes es mucho más alta que en población general (Chi, Sterling, y Weisner, 2006; Hopwood y Grilo, 2010).

Centrándonos en la edad, es destacable que en la muestra del estudio, la edad media de inicio de consumo se sitúa 1.8 años antes que la media de edad de inicio de consumo en población general (Observatorio Español sobre Drogas, 2014). Lo mismo sucede con la edad de patrón regular de consumo, que también se sitúa aproximadamente 1 año antes que en la población de referencia (Observatorio Español sobre Drogas, 2014). Este hecho se explica una vez más por las características clínicas de la muestra, 
ya que son adolescentes con un consumo frecuente y problemático que requiere tratamiento específico (Creemers et al., 2009). Estos resultados ratifican, como se ha descrito ampliamente en la literatura, que una edad de inicio temprana predice un consumo problemático posterior (Martínez-Lorca y Alonso-Sanz, 2003).

Entre perfiles de personalidad no se observan diferencias para la edad de inicio de consumo, la edad de consumo regular y la edad de demanda de tratamiento. La homogeneidad de estos resultados podría estar justificada nuevamente por las características clínicas de la muestra ya que los sujetos han sido reclutados en una unidad específica y comparten aspectos tanto de la cronología del consumo como de la gravedad de la patología adictiva.

Si nos fijamos en el patrón horario de consumo, destaca la frecuencia de consumo en ambos perfiles durante el horario escolar. A su vez, un $69.4 \%$ de la muestra ha repetido curso y un $29.5 \%$ ha abandonado los estudios. Por tanto, el consumo de cánnabis parece estar relacionado significativamente con problemas en el rendimiento académico tal y como se observa en otros estudios (Hall y Degenhardt, 2009; Volkow et al., 2014). Por otra parte, el perfil internalizante reporta más frecuencia de consumo antes de dormir, aspecto que ha sido descrito en otros estudios como uno de los principales efectos buscados en el consumo (Schofield et al., 2006).

La gravedad clínica de ambos perfiles ha sido evaluada con las escalas de preocupaciones expresadas y sindromes clinicos del MACI. En la muestra del estudio, los adolescentes con perfil internalizante obtienen mayores puntuaciones en la mayoría de las escalas en comparación con el perfil externalizante. Centrándonos en el perfil internalizante, los datos observados van en la misma dirección que estudios anteriores (Casullo y Castro, 2002; Fantin, 2006; Szerman et al., 2014). Específicamente, el Afecto depresivo y la Tendencia al suicidio se han descrito como un rasgo muy prevalente en consumidores de sustancias (Chabrol et al., 2014). En contraste, en el perfil externalizante, destacan la Insensibilidad social y la Predisposición a la delincuencia, escalas que se han observado en estudios en los que se comparaban muestras de adolescentes consumidores con muestras de adolescentes no consumidores (Fantin, 2006; Faúndez y Vinet, 2009). Por otro lado, ambos perfiles puntúan alto en Discordancia familiar. Tal y como indica la literatura la presencia de problemática en el seno familiar se relaciona con el consumo de sustancias (Brière et al., 2011). Las puntuaciones más altas obtenidas, independientemente del perfil al cual los adolescentes pertenezcan son: Insensibilidad social, Inclinación al abuso de sustancias, Predisposición a la delincuencia y Propensión a la impulsividad. Como se ha descrito en diversos estudios, los rasgos más disociales e impulsivos están más presentes en población adolescente consumidora (Becoña et al., 2011; Fantin, 2006; Faúndez y Vinet, 2009).
En relación a los efectos subjetivos, ambos perfiles muestran mayor efecto de Euforia, efecto ampliamente descrito en la literatura y más frecuentemente asociado con los motivos de consumo, uso problemático, abuso y dependencia (Block et al., 1998; Scherrer et al., 2009; Zeiger et al., 2010). Por otra parte, el grupo internalizante reporta puntuaciones más elevadas en efectos de Sedación y Disforia. Esto se puede definir, tal y como han descrito Scherrer et al., 2009, como una "Alta Respuesta" al experimentar efectos tanto positivos como negativos. La "Alta respuesta" se ha asociado a una mayor tendencia al consumo y al desarrollo de dependencia (Scherrer et al., 2009). Por tanto, creemos que ante la falta de literatura que relacione personalidad y efectos subjetivos es necesario establecer estudios ulteriores en los que se plantee esta asociación.

Entre las limitaciones del presente estudio encontramos en primer lugar el tamaño reducido de la muestra que limita la potencia estadística de los resultados. En segundo lugar, la muestra del estudio es clínica y por tanto los resultados obtenidos no son extrapolables a población general. Por tanto, la generalización de los resultados a muestras de población de adolescentes consumidores con patrones de uso de cánnabis menos problemáticos debe realizarse con precaución. En tercer lugar, los instrumentos utilizados para la evaluación, MACI y ARCI-49, son auto-administrados y los resultados podrían estar sesgados por una tendencia de los adolescentes a minimizar o maximizar sintomatología. Por último, la escasez y la heterogeneidad de los estudios de personalidad y de los efectos subjetivos del consumo, específicamente en población adolescente consumidora de cánnabis, dificulta la comparación de los resultados obtenidos. No obstante, esto representa a su vez una de las principales aportaciones que hace este trabajo a la literatura existente y, más aún considerando que los resultados de este estudio son consistentes con la investigación empírica y los antecedentes teóricos recabados.

En este estudio se ha observado que la categorización a través de los rasgos de personalidad en dos perfiles -internalizante y externalizante- permite caracterizar una muestra clínica de adolescentes consumidores de cánnabis. La expresión de la gravedad clínica y de los efectos subjetivos es diferente en función de los rasgos de personalidad. Así, el perfil internalizante muestra mayor complejidad clínica; un consumo mayor en horario nocturno, más presencia de preocupaciones expresadas y de síndromes clínicos y mayor experimentación de efectos subjetivos. Conocer el perfil de personalidad internalizante/externalizante es útil para el planteamiento de la intervención con este tipo de población. Discriminar los efectos subjetivos del cánnabis permite conocer las funciones del consumo y plantear el abordaje dirigido a los factores mantenedores de éste.

Como perspectivas futuras y basándonos en los resultados obtenidos, planteamos la necesidad de que se abran nuevas líneas de investigación relacionadas con nuestro 
estudio para dar respuesta a la escasez existente y para ampliar el debate sobre la personalidad y los efectos subjetivos en adolescentes consumidores de sustancias. Si así fuera, apuntar el interés de aumentar el tamaño de la muestra para poder contrastar los resultados obtenidos y aumentar la potencia estadística de los mismos. También, sería deseable protocolizar la evaluación de la personalidad y de los efectos subjetivos de estos adolescentes consumidores, como parte de las valoraciones iniciales para poder diseñar intervenciones más efectivas y ajustadas a las características de estos pacientes.

\section{Conflicto de intereses}

Los autores declaran no tener ningún conflicto de intereses.

\section{Referencias}

Achenbach, T. M. y Edelbrock, C. S. (1984). Psychopathology of childhood. Annual Review of Psychology, 35, 227256. doi:10.1146/annurev.psych.35.1.227

Adshead, G., Brodrick, P., Preston, J. y Deshpande, M. (2012). Personality disorder in adolescence. Advances in Psychiatric Treatment, 18, 109-118. doi:10.1192/apt. bp.110.008623

Alameda-Bailén, J. R., Salguero-Alcañiz, M. P., Merchán-Clavellino, A. y Paíno-Quesada, S. (2014). Mecanismos cognitivos en la toma de decisiones arriesgadas en consumidores de cannabis. Adicciones, 26, 146-158.

Arias, F., Szerman, N., Vega, P., Mesias, B., Basurte, I., Morant, C., ... Babín, F. (2013). Estudio Madrid sobre prevalencia y características de los pacientes con patología dual en tratamiento en las redes de salud mental y de atención al drogodependiente. / Madrid study on the prevalence and characteristics of outpatients with dual pat. Adicciones, 25, 191-200.

Becker, B., Wagner, D., Gouzoulis-Mayfrank, E., Spuentrup, E. y Daumann, J. (2010). The impact of early-onset cannabis use on functional brain correlates of working memory. Progress in Neuro-Psychopharmacology and Biological Psychiatry, 34, 837-845. doi:10.1016/j. pnpbp.2010.03.032

Becoña, E., Fernández del Río, E., Calafat, A. y Fernández-Hermida, J. R. (2014). Attachment and substance use in adolescence: a review of conceptual and methodological aspects. Adicciones, 26, 77-86.

Becoña, E., López, A., Fernádez del Río, E., Martínez, Ú., Fraga, J., Osorio, J., ... Domínguez, M. N. (2011). ¿Tienen una personalidad distinta los adolescentes consumidores de psicoestimulantes? Psicothema, 23, 552-559.

Belcher, A. M., Volkow, N. D., Moeller, F. G. y Ferré, S. (2014). Personality traits and vulnerability or resilience to substance use disorders. Trends in Cognitive Sciences, 18, 211-217. doi:org/10.1016/j.tics.2014.01.010

Block, R. I., Erwin, W. J., Farinpour, R. y Braverman, K. (1998). Sedative, stimulant, and other subjective effects of marijuana: Relationships to smoking techniques. Pharmacology Biochemistry and Behavior, 59, 405-412. doi:10.1016/S0091-3057(97)00453-X

Brière, F. N., Fallu, J. S., Descheneaux, A. y Janosz, M. (2011). Predictors and consequences of simultaneous alcohol and cannabis use in adolescents. Addictive Behaviors, 36, 785-788. doi:10.1016/j.addbeh.2011.02.012

Casullo, M. M. y Castro, A. (2002). Patrones de personalidad, síndromes clínicos y bienestar psicológico en adolescentes. Revista de Psicopatología y Psicología Clínica, 7, 129-140.

Chabrol, H., Melioli, T. y Goutaudier, N. (2014). Association Between Personality Disorders Traits and Problematic Cannabis Use in Adolescents. Substance Use E् Misuse, 141215102232003. doi:10.3109/10826084.2014.984851

Chakroun, N., Doron, J. y Swendsen, J. (2004). Substance use, affective problems and personality traits: Test of two association models. L'Éncephale, 30, 564-569.

Chen, K. W., Banducci, A. N., Guller, L., Macatee, R. J., Lavelle, A., Daughters, S. B. y Lejuez, C. W. (2011). An examination of psychiatric comorbidities as a function of gender and substance type within an inpatient substance use treatment program. Drug and Alcohol Dependence, 118, 92-99. doi:10.1016/j.drugalcdep.2011.03.003

Chi, F. W., Sterling, S. y Weisner, C. (2006). Adolescents with co-occurring substance use and mental conditions in a private managed care health plan: prevalence, patient characteristics, and treatment initiation and engagement. The American Journal on Addictions / American Academy of Psychiatrists in Alcoholism and Addictions, 15, S67-S79. doi:10.1080/10550490601006022

Cosgrove, V. E., Rhee, S. H., Gelhorn, H. L., Boeldt, D., Corley, R. C., Ehringer, M. A., ... Hewitt, J. K. (2011). Structure and etiology of co-occurring internalizing and externalizing disorders in adolescents. Journal of Abnormal Child Psychology, 39, 109-123. doi:10.1007/s10802010-9444-8

Council for International Organizations of Medical Sciences (CIOMS) y World Health Organization (WHO). International Ethical Guidelines for Biomedical Research Involving Human Subjects. Geneva: CIOMS, WHO; 1993. Disponible en: http://www.wma.net/es/30publications/10policies/b3/

Creemers, H. E., Buil, J. M., van Lier, P. A. C., Keijsers, L., Meeus, W., Koot, H. M. y Huizink, A. C. (2015). Early onset of cannabis use: Does personality modify the relation with changes in perceived parental involvement? Drug and Alcohol Dependence, 146, 61-67. doi:10.1016/j. drugalcdep.2014.11.004 
Creemers, H. E., Korhonen, T., Kaprio, J., Vollebergh, W. A. M., Ormel, J., Verhulst, F. C. y Huizink, A. C. (2009). The role of temperament in the relationship between early onset of tobacco and cannabis use: The TRAILS study. Drug and Alcohol Dependence, 104, 113-118. doi:10.1016/j.drugalcdep.2009.04.010

Cuenca-Royo, A. M., Torrens, M., Sánchez-Niubó, A., Suelves, J. M. y Domingo-Salvany, A. (2013). Psychiatric morbidity among young-adults cannabis users. Adicciones, 25, $45-54$.

DuPont, R. L. y Lieberman, J. A. (2014). Young brains on drugs. Science, 344, 557. doi:10.1126/science.1254989

Fantin, M. B. (2006). Perfil de personalidad y consumo de drogas en adolescentes escolarizados. Adicciones, 18, 285-292.

Faúndez, X. y Vinet, E. V. (2009). Tipología Empírica de Adolescentes Consumidores de Drogas según el MACI. Psykhe, 18, 19-35. doi:10.4067/S071822282009000200002

Fergusson, D. M., Horwood, L. J., Lynskey, M. T. y Madden, P. A. F. (2003). Early reactions to cannabis predict later dependence. Archives of General Psychiatry, 60, 10331039. doi:10.1001/archpsyc.60.10.1033

Grant, J. E., Chamberlain, S. R., Schreiber, L. y Odlaug, B. L. (2012). Neuropychological deficits associated with cannabis use in young adults. Drug and Alcohol Dependence, 121, 159-162. doi:10.1016/j.drugalcdep.2011.08.015. Neuropsychological

Gunnarsson, M., Gustavsson, J. P., Tengström, A., Franck, J. y Fahlke, C. (2008). Personality traits and their associations with substance use among adolescents. Personality and Individual Differences, 45, 356-360. doi:10.1016/j. paid.2008.05.004

Guxens, M., Nebot, M., Ariza, C. y Ochoa, D. (2007). Factors associated with the onset of cannabis use: a systematic review of cohort studies. Gaceta Sanitaria, 21, 252260. doi:10.1157/13106811

Hall, W. y Degenhardt, L. (2009). Adverse health effects of non-medical cannabis use. The Lancet, 374, 1383-1391. doi:10.1016/S0140-6736(09)61037-0

Harford, T. C., Chen, C. M., Saha, T. D., Smith, S. M., Ruan, W. J. y Grant, B. F. (2013). DSM-IV Personality disorders and associations with externalizing and internalizing disorders: Results from the national epidemiologic survey on alcohol and related conditions. Journal of Psychiatric Research, 47, 1708-1716. doi:10.1016/j.jpsychires.2013.07.016

Hemphill, S. A., Heerde, J. A., Herrenkohl, T. I., Patton, G. C., Toumbourou, J. W. y Catalano, R. F. (2011). Risk and protective factors for adolescent substance use in Washington State, the United States and Victoria, Australia: A longitudinal study. Journal of Adolescent Health, 49, 312-320. doi:10.1016/j.jadohealth.2010.12.017
Hink, L. K., Rhee, S. H., Corley, R. P., Cosgrove, V. E., Hewitt, J. K., Schulz-Heik, R. J., ... Waldman, I. D. (2013). Personality dimensions as common and broadband-specific features for internalizing and externalizing disorders. Journal of Abnormal Child Psychology, 41, 939-957. doi:10.1007/s10802-013-9730-3

Hopwood, C. J. y Grilo, C. M. (2010). Internalizing and externalizing personality dimensions and clinical problems in adolescents. Child Psychiatry and Human Development, 41, 398-408. doi:10.1007/s10578-010-0175-4

Jacobus, J. y Tapert, S. F. (2014). Effects of cannabis on the adolescent brain. Current Pharmaceutical Design, 20, 2186-93. doi:10.2174/13816128113199990426

Kaufman, J., Birmaher, B., Brent, D., Rao, U., Flynn, C., Moreci, P., ... Ryan, N. (1997). Schedule for Affective Disorders and Schizophrenia for School-Age Children-Present and Lifetime Version (K-SADS-PL): initial reliability and validity data. Journal of the American Academy of Child and Adolescent Psychiatry, 36, 980-988. doi:10.1097/00004583-199707000-00021

Lamas, X., Farré, M., Llorente, M. y Camí, J. (1994). Spanish version of the 49-item short form of the Addiction Research Center Inventory (ARCI). Drug and Alcohol Dependence, 35, 203-9.

Magallón-Neri, E., Díaz, R., Forns, M., Goti, J., Canalda, G. y Castro-Fornieles, J. (2012). Subtypes of adolescents with substance use disorders and psychiatric comorbidity using cluster and discriminant analysis of MMPI-A profiles. Adicciones, 24, 219-228.

Marquez-Arrico, J. E. y Adan, A. (2013). Patología dual y rasgos de personalidad: Situación actual y líneas futuras de trabajo. Adicciones, 25, 195-202.

Martin, W. R., Sloan, J. W., Sapira, J. D. y Jasinski, D. R. (1971). Physiologic, subjective, and behavioral effects of amphetamine, methamphetamine, ephedrine, phenmetrazine, and methylphenidate in man. Clinical Pharmacology and Therapeutics, 12, 245-58.

Martínez-Lorca, M. y Alonso-Sanz, C. (2003). Búsqueda de sensaciones, autoconcepto, asertividad y consumo de drogas ¿Existe relación? Adicciones, 15, 145-157.

Millon, T. (1993). Manual of Millon Adolescent Clinical Inventory. (N. C. Systems., Ed.). Minneapolis.

Millon, T. y Aguirre, G. (2004). MACI: Inventario Clínico para Adolescentes de Millon. (TEA, Ed.). Barcelona.

Munno, D., Saroldi, M., Bechon, E., Sterpone, S. C. y Zullo, G. (2015). Addictive behaviors and personality traits in adolescents. CNS Spectrums, 13, 1-7.

Muro i Rodríguez, A. (2015). Age, sex and personality in early cannabis use. European Psychiatry. doi:10.1016/j.eurpsy.2015.02.008

Newman, J. L. E., Larsen, J. L., Cunningham, K. B. y Barry, R. (2015). An examination of the factor structure of the Millon Adolescent Clinical Inventory in a sample 
of detained adolescent boys. Psychological Assessment, 27, 1022-1036.

Observatorio Español sobre Drogas. (2014). Encuesta Estatal Sobre Uso de Drogas en Enseñanzas Secundarias (ESTUDES). Madrid: Ministerio de Sanidad y Política Social. Delegación del Gobierno para el Plan Nacional sobre Drogas (DGPNSD).

Observatorio Europeo de las Drogas y las Toxicomanias. (2014). Informe Europeo sobre Drogas. Tendencias y novedades. (O. de P. de la U. E. Europea, Ed.). Luxemburgo.

Pedrero Pérez, E. J. (2003). Los trastornos de la personalidad en drogodependientes desde la perspectiva de los cinco grandes factores. Adicciones, 15, 203-220.

Peñafiel, E. (2009). Factores de riesgo y protección en el consumo de sustancias en adolescentes. Pulso, 32, 147173.

Poudevida, S., Farré, M., Roset, P. y Camí, J. (2003). Construcción de un cuestionario para la Valoración de los Efectos Subjetivos de Sustancias con Potencial de Abuso (VESSPA): Evaluación del éxtasis 1. Adicciones, 15, 115126.

Rubino, T., Zamberletti, E. y Parolaro, D. (2012). Adolescent exposure to cannabis as a risk factor for psychiatric disorders. Journal of Psychopharmacology, 26, 177-188. doi:10.1177/0269881111405362

Scherrer, J. F., Grant, J. D., Duncan, A. E., Sartor, C. E., Haber, J. R., Jacob, T. y Bucholz, K. K. (2009). Subjective effects to cannabis are associated with use, abuse and dependence after adjusting for genetic and environmental influences. Drug and Alcohol Dependence, 105, 76-82. doi:10.1016/j.drugalcdep.2009.06.014

Schofield, D., Tennant, C., Nash, L., Degenhardt, L., Cornish, A., Hobbs, C. y Brennan, G. (2006). Reasons for cannabis use in psychosis. Australian and New Zealand Journal of Psychiatry, 40, 570-574. doi:10.1111/j.14401614.2006.01840.x

SPSS, Inc. (1988). SPSS-X User's Guide (3a. ed.). Chicago: Autor

Swift, W., Coffey, C., Degenhardt, L., Carlin, J. B., Romaniuk, H. y Patton, G. C. (2012). Cannabis and progression to other substance use in young adults: findings from a 13-year prospective population-based study. Journal of Epidemiology E Community Health, 66, 1-6. doi:10.1136/jech.2010.129056

Szerman, N., Goti, J., Díaz, R. y Arango, C. (2014). Patología Dual. Protocolos de intervención en adolescentes. (EdikaMed, Ed.). Barcelona.

Ter Bogt, T. F. M., de Looze, M., Molcho, M., Godeau, E., Hublet, A., Kokkevi, A., ... Pickett, W. (2014). Do Societal Wealth, Family Affluence, and Gender account for Trends in Adolescent Cannabis Use? A 30 Country Cross- National StudyNIH Public Access. Addiction, 109, 273-283. doi:10.1016/j.biotechadv.2011.08.021.
Ulloa, R. E., Ortiz, S., Higuera, F., Nogales, I., Fresán, A., Apiquian, R., ... De La Peña, F. (2006). Interrater reliability of the Spanish version of Schedule for Affective Disorders and Schizophrenia for School-Age Children-Present and Lifetime version (K-SADS-PL). Actas Espanolas de Psiquiatria, 34, 36-40. doi:51110615.

United Nation Office on Drugs and Crime. (2014). World drug report. (United Nations, Ed.) Trends in Organized Crime. New York.

Volkow, N. D., Baler, R. D., Compton, W. M. y Weiss, S. R. B. (2014). Adverse health effects of marijuana use. The New England Journal of Medicine, 370, 2219-27. doi:10.1056/ NEJMra1402309

Walther, B., Morgenstern, M. y Hanewinkel, R. (2012). Co-occurrence of addictive behaviours: Personality factors related to substance use, gambling and computer gaming. European Addiction Research, 18, 167-174. doi:10.1159/000335662

Zeiger, J. S., Haberstick, B. C., Corley, R. P., Ehringer, M. a., Crowley, T. J., Hewitt, J. K., ... Rhee, S. H. (2010). Subjective effects to marijuana associated with marijuana use in community and clinical subjects. Drug and Alcohol Dependence, 109, 161-166. doi:10.1016/j.drugalcdep.2009.12.026

Zeiger, J. S., Haberstick, B. C., Corley, R. P., Ehringer, M. A., Crowley, T. J., Hewitt, J. K., ... Rhee, S. H. (2012). Subjective effects for alcohol, tobacco, and marijuana association with cross-drug outcomes. Drug and Alcohol Dependence, 123, S52-S58. doi:10.1016/j.drugalcdep.2012.02.014 